\title{
EQUICONTINUITY OF ITERATES OF AN INTERVAL MAP
}

\author{
A.M. BRUCKNER ${ }^{1}$ AND THAKYIN HU ${ }^{2}$
}

\begin{abstract}
A.bstract. The purpose of this paper is to determine conditions under which equicontinuity of the family of iterates $\left\{f^{n}\right\}$ of a continuous function that maps a. compact interval $I$ into itself does occur. We shall see that this happens only under exceptional circumstances.
\end{abstract}

\section{Introduction}

Let $f$ be a continuous function mapping an interval $I$ into itself. For $n=1,2, \cdots$, let $f^{n+1}=f \circ f^{n}$. Research in the social, biological and physical sciences often leads to a study of the sequence $\left\{f^{n}\right\}$ of iterates [6], [5], [8]. Of importance to the researcher is the question, "if $|x-y|$ is small, will $\left|f^{n}(x)-f^{n}(y)\right|$ be small for all $n$ ?". F or example, if $x_{0}$ denotes the actual initial population of a species of insect and $y_{0}$ the population as estimated by the researcher, one would hope that $\left|f^{n}\left(x_{0}\right)-f^{n}\left(y_{0}\right)\right|$, the error in estimating the population of the $n^{\text {th }}$ generation, would be small if the initial estimate were good. In mathematical language, one would like the family $\left\{f^{n}\right\}$ to be equicontinuous.

Practical problems, however, don't usually lead to equicontinuity of $\left\{f^{n}\right\}$. In fact, one often finds chaotic behavior of various sorts. Theoretically, there could be almost certainly that chaos will arise [3]. In precise language, there is a continuous function $f$ mapping $[0,1]$ into itself and a set $S$ of Lebesque measure 1 such that for $x, y \in S,(x \neq y)$

$$
\begin{gathered}
\lim _{n \rightarrow \infty} \sup \left|f^{n}(x)-f^{n}(y)\right|=1 \\
\lim _{n \rightarrow \infty} \inf \left|f^{n}(x)-f^{n}(y)\right|=0 .
\end{gathered}
$$

Thus, with this function $f$, one can be almost sure that both the true initial value $x_{0}$ and the estimate $y_{0}$ will be in $S$, and using $f^{n}\left(y_{0}\right)$ to predict $f^{n}\left(x_{0}\right)$ is of no value. The function $f$ can be chosen arbitrarily close (uniformly) to the function $g(x)=4 x(1-x)$, a function in the logistic family often arising in practice [6], [7]. We should mention that in practice chaotic situations do arise, but situations in which satisfactory prediction is possible for $x_{0}$ and $y_{0}$ in some set large in measure or category also arise [7].

1 This author was supported in part by a grant from the NSF.

2 This author was supported in part by a grant from the NSC of R.O.C.

Received November 2, 1987, revised August 13, 1990. 
The purpose of this article is to determine conditions under which the ideal situation, equicontinuity of the family of iterates, does occur. We shall see that this happens only under exceptional circumstances. Our main results are summarized in Theorem 11.

\section{Main Results}

We begin with the following lemma.

Lemma 1. Let $f: I \rightarrow I$ be a continuous mapping of a compact interval I into itself. Suppose the fixed point set of $f$ is $[a, b]$ with $a<b$. Then $(i) x<a$ implies $f(x)>x$ and (ii) $x>b$ implies $f(x)<x$.

Proof. By hypothesis, if $x \notin[a, b]$, then $f(x) \neq x$. Assume (i) is not true, then there exists $x<a$ with $f(x) \leq x$. On the other hand $f(0)>0$. It follows then from the Intermediate Value Theorem that there exists some $c \in(0, x)$ with $f(c)=c$ which is a contradiction. Hence (i) is true. Similarly, (ii) is proved.

Lemma 2. Let $f: I \rightarrow I$ be a continuous mapping of a compact interval I into $I$ with fixed point set $[a, b]$, where $a<b$. Then there exists some interval $(\alpha, \beta)$ containing $[a, b]$ such that for each $x \in(\alpha, \beta) \cap I$, the sequence of iterates $\left\{f^{n}(x)\right\}$ converges to a fixed point of $f$.

Proof. For the positive number $\varepsilon=b-a$, we may use the continuity of $f$ and the fact that $f(a)=a$ to get $\delta>0$ such that $x \in(a-\delta, a+\delta)$ implies $|f(x)-f(a)|=|f(x)-a|<\varepsilon$, i.e. $f(x) \in(a-\varepsilon, a+\varepsilon)=(a-\varepsilon, b)$. Now either $f(x) \in[a, b)$ or $f(x) \in(a-\varepsilon, a)$. If $f(x) \in[a, b)$, we are done. If $f(x) \in(a-\varepsilon, a)$, then Lemma 1 implies $f(x)>x$ which implies $f(x) \in(a-\delta, a+\delta)$ and which in turn implies $f^{2}(x) \in(a-\varepsilon, b)$. Inductively, either there exists some $N$ such that either $f^{N}(x) \in[a, b)$ (in this case we are done) or $f^{n}(x)<a$ for all $n$ with $x<f(x)<f^{2}(x)<\cdots$. In the latter case, $\left\{f^{n}(x)\right\}$ is an increasing sequence of real numbers bounded above and hence $\lim _{n \rightarrow \infty} f^{n}(x)$ exists. Clearly this limit is a fixed point of $f$, in fact, the limit is $a$. Thus, we have shown that for all $x \in(a-\delta, a+\delta)$, the sequence of iterates $\left\{f^{n}(x)\right\}$ converges to a fixed point of $f$. Similarly, we may prove that there exists some $\delta^{\prime}>0$ such that for all $x \in\left(b-\delta^{\prime}, b+\delta^{\prime}\right)$, the sequence of iterates $\left\{f^{n}(x)\right\}$ converges to a fixed point of $f$.

We shall also make use of the following lemma which is provable by the same techniques as above.

Lemma 3. Let $f: I \rightarrow I$ be a continuous mapping of a compact interval I into itself with fixed point set $[a, b]$, where $a<b$. Then for any $\varepsilon>0$, there exists $\delta$ with $0<\delta<\varepsilon$ such that $|y-a|<\delta$ implies $\left|f^{n}(y)-a\right|<\varepsilon$ for all $n$.

Our next result provides some sufficient conditions for $\left\{f^{n}\right\}$ to be equi-continuous.

Proposition 4. Let $f: I \rightarrow I$ be a continuous mapping such that its fixed point set is a non-degenerate interval. Suppose that for each $x$ in $I,\left\{f^{n}(x)\right\}$ converges to a fixed 
point of $f$. Then $\left\{f^{n}\right\}$ is equi-continuous.

Proof. Let the fixed point set of $f$ be $[a, b]$ with $a<b$ and let $x \in I$ be given. Since $\left\{f^{n}(x)\right\}$ converges to a fixed point of $f$, we need to consider the following three cases: (i) there exists some $N$ such that $f^{N}(x) \in(a, b)$, (ii) $f^{n}(x) \notin(a, b)$ for any $n$ with $\lim _{n \rightarrow \infty} f^{n}(x)=a$, and (iii) $f^{n}(x) \notin(a, b)$ for any $n$ with $\lim _{n \rightarrow \infty} f^{n}(x)=b$. In case (i), for $\varepsilon=\min \left\{\left|f^{N}(x)-a\right|,\left|f^{N}(x)-b\right|\right\}>0$, we may use the equi-continuity of $\left\{f^{1}, f^{2}, \cdots, f^{N}\right\}$ to get a $\delta>0$ such that $|y-x|<\delta$ implies $\left|f^{i}(y)-f^{i}(x)\right|<\varepsilon$ for $i=1,2, \cdots, N$. Observe that $f^{N}(y) \in(a, b)$. Consequently $\left|f^{n}(y)-f^{n}(x)\right|=\mid f^{N}(y)-$ $f^{N}(x) \mid<\varepsilon$ for $n \geq N$. Thus $\left\{f^{n}\right\}_{n=1}^{\infty}$ is equicontinuous at $x$. In case (ii), suppose $\varepsilon>0$ is given. First we apply Lemma 3 to get some $\varepsilon^{\prime}$ with $0<\varepsilon^{\prime}<\frac{\varepsilon}{2}$ such that

$$
|y-a|<\varepsilon^{\prime} \quad \text { implies } \quad\left|f^{n}(y)-a\right|<\frac{\varepsilon}{2} \quad \text { for all } n \text {. }
$$

Next, $\lim _{n \rightarrow \infty} f^{n}(x)=a$ yields an $N$ such that $n \geq N$ implies $\left|f^{n}(x)-a\right|<\varepsilon^{\prime} / 2$. Also, we may use the equi-continuity of $\left\{f, f^{2}, \cdots f^{N}\right\}$ at $x$ to get a $\delta>0$ such that $|y-x|<\delta$ implies $\left|f^{i}(y)-f^{i}(x)\right|<\varepsilon^{\prime} / 2$ for $i=1,2, \cdots, N$. In particular, $|y-x|<\delta$ implies $\left|f^{N}(x)-f^{N}(y)\right|<\varepsilon^{\prime} / 2$. Thus $\left|f^{N}(y)-a\right| \leq\left|f^{N}(y)-f^{N}(x)\right|+\left|f^{N}(x)-a\right|<$ $\varepsilon^{\prime} / 2+\varepsilon^{\prime} / 2=\varepsilon^{\prime}$. It follows now from (1) that $\left|f^{n}(y)-a\right|<\varepsilon / 2$ for all $n \geq N$. Consequently for $n \geq N$, we get

$$
\begin{aligned}
\left|f^{n}(y)-f^{n}(x)\right| & \leq\left|f^{n}(y)-a\right|+\left|a-f^{n}(x)\right| \\
& <\frac{\varepsilon}{2}+\frac{\varepsilon^{\prime}}{2}<\frac{\varepsilon}{2}+\frac{\varepsilon}{2}=\varepsilon .
\end{aligned}
$$

Thus, we have shown that for any $\varepsilon>0$, there exists $\delta>0$ such that $|y-x|<\delta$ implies $\left|f^{n}(y)-f^{n}(x)\right|<\varepsilon$ for all $n$, i.e., $\left\{f^{n}\right\}$ is equi-continuous at $x$.

Conversely, we have the following result:

Proposition 5. Let $f: I \rightarrow I$ be a mapping such that $\left\{f^{n}\right\}$ is equicontinuous. Suppose $f$ has more than one fixed point. Then for each $x$ in $I,\left\{f^{n}(x)\right\}$ converges to a fixed point of $f$.

Proof. Equicontinuity of $\left\{f^{n}\right\}$ implies that the fixed point set $F$ of $f$ is connected and closed. Since $F$ is not a singleton, $F$ must be a non-degenerate interval, say $F=[\alpha, \beta]$ with $\alpha<\beta$. Assume $0<\alpha<\beta<1$, we claim that there exists $\alpha_{1}, \beta_{1}$ with $[\alpha, \beta] \subseteq$ $\left(\alpha_{1}, \beta_{1}\right)$ such that $x \in\left(\alpha_{1}, \beta_{1}\right)$ implies $f^{n}(x)$ converges to a fixed point of $f$. Note that, by Lemma $1, x<\alpha$ implies that $f(x)>x$. We then use the continuity of $f$ and the fact that $f(\alpha)=\alpha$ to get a $\delta$ with $0<\delta<(\beta-\alpha)$ such that $x \in(\alpha-\delta, \alpha+\delta)$ implies $f^{n}(x)<\beta$. Let $\alpha_{1}=\alpha-\delta$. Then it is easy to see that $x \in\left(\alpha_{1}, \beta\right]$ implies $f^{n}(x)$ converges to a fixed point of $f$. Similarly, we may find a $\beta_{1}>\beta$ such that $x \in\left[\alpha, \beta_{1}\right)$ implies that $\left\{f^{n}(x)\right\}$ converges to a fixed point of $f$ and the claim is proved. Next, let $K=\left\{x \in I: f^{n}(x)\right.$ converges to a fixed point of $f\}$. Firstly, $K \neq \emptyset$ since $K \supseteq[\alpha, \beta]$. Secondly, we show that $K$ is open. For that purpose, let $x \in K$ and a positive number $\varepsilon<\min \left(\frac{\alpha-\alpha_{1}}{2}, \frac{\beta_{1}-\beta}{2}\right)$ 
be given. Since $f^{n}(x)$ converges to a fixed point of $f$, there exists an integer $N$ such that $n \geq N$ implies that $f^{n}(x) \in(\alpha-\varepsilon, \beta+\varepsilon)$. Also equicontinuity of $\left\{f^{n}\right\}$ implies that there exists a neighborhood $U(x)$ such that $y \in U(x)$ implies $\left|f^{n}(y)-f^{n}(x)\right|<\varepsilon$. Thus $y \in U(x)$ implies $f^{N}(y) \in\left(\alpha_{1}, \beta_{1}\right)$. It follows now from our earlier claim that $f^{n}(y)$ converges to a fixed point of $f$. Thirdly, we show that $K$ is also closed. For that purpose, use equi-continuity of $\left\{f^{n}\right\}$ on $I$ to find a $\delta>0$ such that $|x-y|<\delta$ implies $\left|f^{n}(x)-f^{n}(y)\right|<\varepsilon<\min \left(\frac{\alpha-\alpha_{1}}{2}, \frac{\beta_{1}-\beta}{2}\right)$ for all $n$. Now suppose $\lim _{n \rightarrow \infty} x_{n}=x$ with $x_{n} \in K$. Choose $m$ large enough such that $\left|x_{m}-x\right|<\delta$. For this $x_{m}$, we may choose $N$ such that $f^{N}\left(x_{m}\right) \in(\alpha-\varepsilon, \beta+\varepsilon)$. Thus $\left|f^{N}(x)-f^{N}\left(x_{m}\right)\right|<\varepsilon$ which in turn implies that $f^{N}(x) \in\left(\alpha_{1}, \beta_{1}\right)$ and consequently $f^{n}(x)$ converges to a fixed point of $f$ and the proof is complete.

It was convenient to state Propositions 4 and 5 as converses, but this required the condition that the fixed point set for $f$ be a nondegenerate interval. That the fixed point set for $f$ be connected is clearly necessary for equi-continuity of the set $\left\{f^{n}\right\}$. (If $f(a)=a<b=f(b)$ but $f(x)>x($ or $f(x)<x)$ for all $x \in(a, b)$, then $\left(f^{n}\right)^{-1}\left(\frac{a+b}{2}\right)$ contains points arbitrarily close to a (or to $b)$ ).

We turn to a brief discussion of what can happen when the fixed point set is a degenerate interval $\{c\}$. Observe first that the function $f(x)=1-x$ satisfies $f^{2}(x)=x$ for all $x$. Thus $\left\{f^{n}\right\}$ is equi-continuous but $\left\{f^{n}(x)\right\}$ does not converge unless $x=\frac{1}{2}$, the fixed point. Thus Proposition 5 fails without the nondegeneracy assumption. On the other hand, Proposition 4 remains valid when $f$ has a single fixed point.

Proposition 6. Let $f$ be a continuous function mapping $I_{0}$ into $I_{0}$. If for each $n=1,2, \cdots, c$ is the only fixed point for $f^{n}$, then $\left\{f^{n}\right\}$ is equicontinuous. In particular, this occurs if $f^{n}(x) \rightarrow c$ for all $x$, where $c$ is the unique fixed point of $f$.

Proof. It follows readily from the assumption that $c$ is the only fixed point for each $f^{n}$ that for all $n$,

$$
\begin{aligned}
& f^{n}(x)>x \text { for all } x \in[0, c) \\
& \text { and } f^{n}(x)<x \text { for all } x \in(c, 1] \text {. }
\end{aligned}
$$

We use these facts to show that if $J$ is any interval in $I_{0}$ such that $f(J) \subset J$, then $f(J) \subset \operatorname{Int}(J)$. This in turn, will imply that the sequence $\left\{f^{n}\right\}$ converges uniformly to $c$, from which equicontinuity of $\left\{f^{n}\right\}$ will follow from Ascoli's Theorem.

Suppose $J=[a, b]$ and $f(J) \subset J$. It is clear from $\left({ }^{*}\right)$ that $c \in \operatorname{Int}(J)$. Choose $x_{1} \in J$ such that $f\left(x_{1}\right)=a$. Then $x_{1}>c$ because of $\left(^{*}\right)$. Inductively, obtain a sequence $\left\{x_{n}\right\}_{1}^{\infty}$ such that $f\left(x_{n+1}\right)=x_{n}$. Then $x_{n}>x_{n-1}$ for all $n=1,2, \cdots$. To see this, observe that if for some $n, x_{n+1}<x_{n}$, then $x_{n}<c$ by $\left(^{*}\right)$. But then $f^{n+1}\left(x_{n+1}\right)=a$, which is impossible, again by $\left({ }^{*}\right)$. Thus $x_{n}$ increases to an element $x_{\infty}$ and $f\left(x_{\infty}\right)=x_{\infty}$. This is impossible since $c$ is the only fixed point of $f$. Thus $a \notin f(J)$. Similarly, $b \notin f(J)$. Consequently $f(J) \subseteq \operatorname{Int}(J)$. 
Next, let $I_{1}=f\left(I_{0}\right)$ and for each $n=2,3, \cdots$, let $I_{n+1}=f\left(I_{n}\right)$. Write $I_{n}=\left[a_{n}, b_{n}\right]$. It is clear that either $I_{n}=\{c\}$ for some $n$ or that $I_{n+1} \subset \operatorname{Int}\left(I_{n}\right)$ for all $n$ so that $\left\{a_{n}\right\}$ is an increasing sequence and $\left\{b_{n}\right\}$ is a decreasing sequence. Let $a=\lim _{n \rightarrow \infty} a_{n}, b=$ $\lim _{n \rightarrow \infty} b_{n}$. Then $f([a, b])=[a, b]$. But this implies $a=b=c$. It follows that for each $\varepsilon>0$, there exists a positive integer $n$ such that $f^{n}\left(I_{0}\right) \subset(c-\varepsilon, c+\varepsilon)$; in other words, $f^{n} \rightarrow c$ uniformly. The equicontinuity of $\left\{f^{n}\right\}$ is now an immediate consequence of Ascoli's Theorem.

For a mapping of an interval $I$ onto itself, there is a particularly simple necessary and sufficient condition for equi-continuity of the sequence $\left\{f^{n}\right\}$ : namely that $f^{2}$ be the identity. That the condition is sufficient is obvious. The necessity will follow as a corollary to the following proposition.

Proposition 7. Let $(X ; d)$ be a compact metric space and $f: X \rightarrow X$ be a surjective mapping whose sequence of iterates $\left\{f^{n}\right\}$ is equi-continuous. Then for any two points $x, y$ in $X$, the two sequences of iterates $\left\{f^{n}(x)\right\},\left\{f^{n}(y)\right\}$ have convergent subsequences of the same indices $\left\{f^{n_{i}}(x)\right\},\left\{f^{n_{i}}(y)\right\}$ such that $f^{n_{i}}(x) \rightarrow x, f^{n_{i}}(y) \rightarrow y$ as $i \rightarrow \infty$.

Proof. Let $x_{0}, y_{0}$ be two arbitrary points of $X$. Define $x_{n}, y_{n}$ by $f\left(x_{n+1}\right)=$ $x_{n}, f\left(y_{n+1}\right)=y_{n}$ for $n=0,1,2, \cdots$. We may assume without loss of generality that $\left\{x_{n}\right\},\left\{y_{n}\right\}$ have convergent subsequences with the same subscripts, say $\left\{x_{n_{k}}\right\},\left\{y_{n_{k}}\right\}$ such that $x_{n_{k}} \rightarrow x_{\infty}, y_{n_{k}} \rightarrow y_{\infty}$ as $k \rightarrow \infty$. Uniform equi-continuity of $\left\{f^{n}\right\}$ yields a $\delta>0$ such that $d(x, y)<\delta$ implies $d\left(f^{n}(x),\left(f^{n}(y)\right)<1\right.$ for all $n$. For this $\delta>0$, we may choose an $N$ such that $k \geq N$ implies $d\left(x_{n_{k}}, x_{n_{(k+i)}}\right)<\delta, d\left(y_{n_{k}}, y_{n_{(k+i)}}\right)<\delta$ for $i=1,2, \cdots$. Fix $k \geq N$ and define $p_{i}=n_{(k+i)}-n_{k}$ for $i=1,2, \ldots$. Thus $p_{1}<p_{2}<\ldots$, and $p_{i} \rightarrow \infty$ as $i \rightarrow$ $\infty$. Now $d\left(x_{n_{k}+p_{i}}, x_{n_{k}}\right)<\delta$ implies $d\left(x_{0}, f^{p_{i}}(x)\right)=d\left(f^{n_{k}+p_{i}}\left(x_{n_{k}+p_{i}}\right), f^{n_{k}+p_{i}}\left(x_{n_{k}}\right)\right)<1$ for all $p_{i}$. Similarly, $d\left(y_{0}, f^{p_{i}}\left(y_{0}\right)\right)<1$ for all $p_{i}$. Let one such $p_{i}$ be called $m_{1}$ amd we have $d\left(x_{0}, f^{m_{1}}\left(x_{0}\right)\right)<1, d\left(y_{0}, f^{m_{1}}\left(y_{0}\right)\right)<1$. By the same procedure there exist $\left\{f^{q_{i}}\left(x_{0}\right)\right\},\left\{f^{q_{i}}\left(y_{0}\right)\right\}$ such that

$$
d\left(x_{0}, f^{q_{i}}\left(x_{0}\right)\right)<\frac{1}{2}, d\left(y_{0}, f^{q_{i}}\left(y_{0}\right)\right)<\frac{1}{2} \quad \text { for } \quad i=1,2, \cdots,
$$

where $q_{i} \rightarrow \infty$ as $i \rightarrow \infty$. Thus we may choose some $q_{i}>m_{1}$ and call it $m_{2}$. Inductively, we get $\left\{f^{m_{j}}\left(x_{0}\right)\right\},\left\{f^{m_{j}}\left(y_{0}\right)\right\}$ such that

$$
d\left(x_{0}, f^{m_{j}}\left(x_{0}\right)\right)<\frac{1}{j}, d\left(y_{0}, f^{m_{j}}\left(y_{0}\right)\right)<\frac{1}{j}, \quad \text { for } \quad j=1,2, \cdots .
$$

Hence $f^{m_{j}}\left(x_{0}\right) \rightarrow x_{0}, f^{m_{j}}\left(y_{0}\right) \rightarrow y_{0}$.

Corollary 8. Let $(X, d)$ be a compact metric space and $f: X \rightarrow X$ be a surjective mapping whose sequence of iterates $\left\{f^{n}\right\}$ is equicontinuous. Then $f$ is a homeomorphism. (This corollary has also been obtained by Young [9] in connection with work of a different nature). 
Proof. It suffices to show that $f$ is injective, a fact that follows immediately from the previous result.

Corollary 9. Let $J=[a, b]$ be an interval. If $f$ maps $J$ onto $J$ and $\left\{f^{n}\right\}$ is equicontinuous, then $f^{2}$ is the identity.

Proof. The function $f$ is a homeomorphism of $J$ onto $J$. If $f(a)=a$, then $f(b)=b$. Since the fixed point set of $f$ must be connected, $f$ is the identity. If $f(a)=b$, then $f(b)=a$. Thus $f^{2}(a)=a, f^{2}(b)=b$ and since the fixed point set for $f^{2}$ is connected, $f^{2}$ is the identity.

Corollary 10. If $f$ maps $I_{0}$ into $I_{0}$ and $\left\{f^{n}\right\}$ is equi-continuous, then there exists an interval $E=[a, b]$ such that $f^{2}$ is the identity on $E$. Furthermore for $x \notin E, f^{2 n}(x)$ converges to some point of $E$. The interval $E$ will be nondegenerate unless $f^{n}$ has a unique fixed point for all $n$.

Proof. Let $E=\cup_{n=1}^{\infty} E_{n}$, where $E_{n}$ is the fixed point set for $f^{n}$. Then each $E_{n}$ is connected. Suppose $E_{n}$ is nondegenerate for some $n$, Then $E$ is connected. In fact, if $x, y \in E$, then there exist $m, n$ such that $x \in E_{m}, y \in E_{n}$ so $x$ and $y$ are in $E_{n m}$ and $[x, y] \subseteq E$. Let $[a, b]$ be the smallest interval containing $E$. Since $E$ consists of the periodic points of $f, f$ maps $E$ onto $E$ and consequently $f$ maps $[a, b]$ onto $[a, b]$. It follows from Corollary 9 that $f^{2}$ is the identity on $[a, b]$. If $x \notin E$, then by Proposition $5, f^{2 n}(x)$ converges to some point of $E$.

Remark. We cannot conclude that $f^{n}(x)$ converges to some point of $E$, but $f^{n}(x)$ is attracted to $E$ in the sense that $\operatorname{dist}\left(f^{n}(x), E\right) \rightarrow 0$ as $n \rightarrow \infty$. The function

$$
f(x)=\left\{\begin{array}{lll}
-\frac{1}{2}\left(x-\frac{1}{4}\right)+\frac{3}{4}, & \text { if } & 0 \leq x \leq \frac{1}{4} \\
1-x & \text { if } & \frac{1}{4} \leq x \leq \frac{3}{4} \\
-\frac{1}{2}\left(x-\frac{3}{4}\right)+\frac{1}{4} & \text { if } & \frac{3}{4} \leq x \leq 1
\end{array}\right.
$$

furnishes an example, as straightforward computations show.

Finally, we summarize the results of this section as the following theorem.

Theorem 11. Let $f: I \rightarrow I$ be a continuous mapping of a compact interval I into itself.

(1) If the fixed point set of $f$ is connected and for each $x,\left\{f^{n}(x)\right\}$ converges to a fixed point of $f$, then $\left\{f^{n}\right\}$ is equicontinuous.

(2) If $\left\{f^{n}\right\}$ is equicontinuous, then for each $x$ in $I$, $\operatorname{dist}\left(f^{n}(x), E\right) \rightarrow 0$ as $n \rightarrow \infty$, where $E$ is the fixed point set of $f^{2}$. Furthermore, if $f$ has more than one fixed point, then for each $x$ in $I,\left\{f^{n}(x)\right\}$ converges to a fixed point of $f$.

(3) If $\left\{f^{n}\right\}$ is equicontinuous and $f$ is surjective, then $f$ is a homeomorphism and $f^{2}$ is the identity mapping. 


\section{Additional Remarks.}

We close with some simple remarks related to the work of the previous section.

Corollary 12. Let $f: I \rightarrow I$ be a continuous mapping. Then $\left\{f^{n}\right\}$ is equicontinuous if and only if $\cap_{n=1}^{\infty} f^{n}(I)=F_{2}$, where $F_{2}$ is the fixed point set of $f^{2}$.

Proof. First, observe that $f: \cap_{n=1}^{\infty} f^{n}(I) \rightarrow \cap_{n=1}^{\infty} f^{n}(I)$ is onto and $\cap_{n=1}^{\infty} f^{n}(I)$ is an interval. It follows then from the equicontinuity of $\left\{f^{n}\right\}$ and Corollary 8 that $\cap_{n=1}^{\infty} f^{n}(I)=F_{2}$. Conversely, since $F_{2}=\cap_{n=1}^{\infty} f^{n}(I)$ is connected, it follows from a result of $W$. Boyce [1] that $\left\{f^{n}\right\}$ is equicontinuous.

The following result due to $J$. Cano [4] also follows immediately from Proposition 5.

Corollary 13. Let $f: I \rightarrow I$ be a mapping such that $\left\{f^{n}\right\}$ is equicontinuous. If the fixed point set of $f$ is non-degenerate, then the set of periodic points of $f$ coincides with the fixed point set of $f$.

Combining Corollaries 12 and 13 , we see that when $\left\{f^{n}\right\}$ is equicontinuous, there can be periodic points of order one or two, but of no other orders. This may sometimes provide a quick proof that a particular family is not equicontinuous. For reference, we state this as a corollary.

Corollary 14. Let $f: I \rightarrow I$ be a continuous mapping. If $f$ possesses a point of minimal period $n \geq 2$, then $\left\{f^{n}\right\}$ cannot be equicontinuous.

Remark. A continuous mapping $f: I \rightarrow I$ is said to be expanding if $I$ can be divided into finitely many subintervals $I_{1}, I_{2}, \ldots, I_{n}$. Such that there exists $\lambda>1$ with $|f(x)-f(y)| \geq \lambda|x-y|$ for all $x, y$ belonging to the same subinterval $I_{j}$. These mappings have been studied by Byers [2] and Zhang [10]. They proved that such a mapping possesses a periodic point whose period is not an integral power of 2 . It follows [2] that $f$ is chaotic. One might suspect that the expanding constant $\lambda>1$ can be relaxed to 1 on some subinterval $I_{k}$. We give the following easy example to show it cannot.

Example. Let $[a, b] \subseteq(0,1) \subseteq[0,1]=I$. We may define a piecewise linear mapping $f: I \rightarrow I$ in the following manner. If $x \in[a, b]$, define $f(x)=x$; if $0 \leq x<a$, define $f(x)$ in a rather arbitrary manner except that $x<f(x)<b$ and the slopes of the line segments of its graph is larger in absolute value than some given $\lambda>1$; if $b<x \leq 1$, define $f(x)$ such that $a<f(x)<x$ with the corresponding slopes larger than $\lambda$. Then it is easy to see that for each $x$ in $I, f^{n}(x)$ converges to a fixed point of $f$ and hence $f$ does not possess any periodic points other than the fixed points.

\section{References}

[1] W. Boyce, "Г-compact maps on an interval and fixed points", Trans. Amer. Math. Soc. 169 (1971), 82-102. 
[2] B. Byers, "Periodic points and chaos for expanding maps of the interval", Bull. Austral. Math. Soc. 24. (1981), 79-83.

[3] A. Bruckner and T. Hu, "On scrambled sets for chaotic functions", Trans. Amer. Math. Soc. Vol 301, 289-297, 1987.

[4] J. Cano, "Common fixed points for a class of commuting mappings on an interval", Proc. Amer. Math. Soc. 86, No 2 (1982), 336-338.

[5] T. Li and J. Yorke, "Period three implies chaos", Amer. Math. Monthly 82 (1975), 985-992.

[6] R. May, "Simple mathematical models with very complicated dynamics", Nature, 261 (1976), 459 467.

[7] C. Preston, "Iterates of maps on an interval", Lecture notes in Mathematics 999, Springer 1983.

[8] E. Vul, Y. Sinai, and K. Khanin, "Felgenbaum univerality and the thermodynamic formalism", Russian Math. Surveys, 39 (1984), 1-40.

[9] S. Young, "Concerning the function equation $f(g)=f$, regular mappings and periodic mappings", (to appear).

[10] Z. Zhang, "Periodic points and chaos for expanding self-maps of the interval", Bull. Austral. Math. Soc. 31 (1985), 439-443.

\author{
A.M. Bruckner \\ Department of Mathematice, University of California, Santa Barbara, California, 93106 USA. \\ Thakyin Hu \\ Department of Mathematics, Tamkang University, Tamsui, Taiwan,R.O.C.
}

